
Cahiers
de a Recherche
Furles Droits
Fondamentaux

\section{Cahiers de la recherche sur les droits} fondamentaux

16 | 2018

Les partis politiques

\title{
L'Union européenne et le Sahara occidental : pas (seulement) une affaire de droits de l'homme
}

Commentaire sous l'arrêt Western Sahara Campaign de la CJUE du 27 février 2018

The European Union and the Western Sahara: Not (Only) a Case of Human

Rights. Comments on the Western Sahara Campaign Judgement by the CJEU of

February 27, 2018

Carlos Ruiz Miguel

\section{(2) OpenEdition}

\section{Journals}

Édition électronique

URL : https://journals.openedition.org/crdf/327

DOI : $10.4000 /$ crdf.327

ISSN : 2264-1246

Éditeur

Presses universitaires de Caen

\section{Édition imprimée}

Date de publication : 16 novembre 2018

Pagination : 123-140

ISBN : 978-2-84133-901-3

ISSN : $1634-8842$

Référence électronique

Carlos Ruiz Miguel, «L'Union européenne et le Sahara occidental : pas (seulement) une affaire de droits de l'homme », Cahiers de la recherche sur les droits fondamentaux [En ligne], 16 | 2018, mis en ligne le 16 novembre 2019, consulté le 14 novembre 2022. URL : http://journals.openedition.org/crdf/ 327 ; DOI : https://doi.org/10.4000/crdf.327 


\title{
L'Union européenne et le Sahara occidental: pas (seulement) une affaire de droits de l'homme
}

\section{Commentaire sous l'arrêt Western Sahara Campaign de la CJUE du 27 février 2018}

\author{
Carlos RUIZ MIGUEL \\ Professeur de droit constitutionnel à l'université de Saint-Jacques de Compostelle (Espagne)
}

I. Le statut du Sahara occidental et la question des droits de I'homme au niveau des Nations unies
A. Le statut international du Sahara occidental
B. La question des droits de I'homme dans les résolutions du Conseil de sécurité sur le Sahara occidental

II. La position du Parlement européen sur le statut international du Sahara occidental et les droits de l'homme
A. Le statut du Sahara occidental dans les résolutions du Parlement européen
B. Les droits de l'homme au Sahara occidental et le Parlement européen

III. La question du Sahara occidental dans les avis du service juridique du Parlement européen
A. L'avis de 2006
B. L'avis de 2009
C. L'avis de 2011
D. L'avis de 2013

IV. Statut juridique du Sahara occidental et droits de l'homme dans la jurisprudence de la CJUE

A. L'affaire dite Polisario (T-512/12 et C-104/16 P)

1. La demande du Front Polisario

2. L'arrêt de la $8^{e}$ chambre de la CJUE du 10 décembre 2015: les droits de l'homme comme critère décisif

3. Le pourvoi en appel et l'opinion de l'avocat général du 13 septembre 2016

4. L'arrêt de la grande chambre de la CJUE du 21 décembre 2016

B. L'affaire dite Western Sahara Campaign (C-266/16)

1. La question préjudicielle de la High Court of Justice (England \& Wales)

2. L'opinion de l'avocat général du 10 janvier 2018

3. L'arrêt de la grande chambre de la CJUE du 27 février 2018

V. Le Conseil récidive alors que la Cour enfonce le clou 
L'arrêt dit Western Sahara Campaign (C-266/16) n'est pas la première décision de la Cour de justice de l'Union européenne (CJUE) concernant le Sahara occidental, mais elle revêt une importance particulière parce qu'elle consolide une doctrine déjà établie dans son précédent arrêt Polisario du 21 décembre 2016 (C-104/16 P). La grande chambre de la CJUE va trancher dans les deux affaires les questions posées autour du Sahara occidental sans faire référence dans son argumentation aux droits de l'homme. Ceci ne veut toutefois pas dire que l'exigence du respect des droits de l'homme a été ou devra être ignorée dans les relations de l'Union européenne (UE) avec les acteurs internationaux concernés (Maroc, Front Polisario).

\section{Le statut du Sahara occidental et la question des droits de l'homme au niveau des Nations unies}

\section{A Le statut international du Sahara occidental}

Le Sahara occidental ${ }^{1}$, d'après les Nations unies, est un «territoire non autonome» qui attend encore le parachèvement de sa décolonisation. Comme tel, il est inscrit dans l'agenda de la Quatrième Commission (Politiques spéciales et décolonisation $)^{2}$. La décolonisation du territoire doit être opérée par «l'expression libre et authentique de la volonté des populations du territoire $»^{3}$, soit à travers un référendum d'autodétermination comme l'ont exigé les résolutions de l'Assemblée générale ${ }^{4}$ et du Conseil de sécurité 5 . Le Conseil de sécurité avait publié un Plan de règlement agréé par les deux parties au conflit (le Royaume du Maroc et le Front Polisario $\left.{ }^{6}\right)^{7}$ qui fut complété par des nouveaux accords entre les deux parties adoptés en 1997 (accords de Houston ${ }^{8}$ ) et 19999, approuvés par le Conseil de sécurité ${ }^{10}$. À cette fin, le Conseil de sécurité avait instauré la mission des Nations unies pour l'organisation d'un référendum au Sahara occidental (MINURSO) ${ }^{11}$, qui avait pu achever le recensement de la liste des votants au référendum en décembre 1999, après avoir surmonté nombre d'obstacles $^{12}$. Après cette finalisation du recensement, et au vu et su de la composition de la liste électorale (qui, avec peu de doutes, vouait à l'échec l'option d'intégration du territoire au Royaume du Maroc), le Royaume du Maroc décida de ne plus honorer ses engagements. Quelques mois après la communication de la liste des votants aux deux parties au conflit, le Maroc recommençait à poser des obstacles au processus, pour finalement communiquer au secrétaire général des Nations unies en avril 2004 (après un brutal bouleversement de la situation politique en Espagne) son intention de ne plus poursuivre l'application du Plan de règlement, préalablement accepté par les deux parties (Maroc et Front Polisario) et approuvé par le Conseil de sécurité, en déclarant que «Le caractère définitif de la solution d'autonomie n'est [...] pas négociable pour le Royaume» ${ }^{13}$.

\section{B. La question des droits de l'homme dans les résolutions du Conseil de sécurité sur le Sahara occidental ${ }^{14}$}

Le blocage du processus politique pour l'organisation du référendum a permis, au niveau des Nations unies, de soulever d'autres questions éclipsées par le référendum, et notamment la question des droits de l'homme et la question de la légalité des activités économiques se développant dans le territoire. À cet égard, il faut rappeler que le plan Baker présenté en 2003 suite au blocage du Plan de règlement ${ }^{15}$ contenait déjà des éléments sur les

1. Parmi les ouvrages en langue française qui traitent avec le plus de rigueur cette question, voir M. de Froberville, Sahara occidental. La confiance perdue, Paris, L'Harmattan, 1996; Le droit international et la question du Sahara occidental, V. Chapaux, K. Arts, P. Pinto Leite (dir.), Porto, International Platform of Jurists for East Timor, 2009.

2. La question du Sahara occidental est inscrite dans la «Liste préliminaire annotée des questions à inscrire à l'ordre du jour provisoire de la soixante-douzième session ordinaire de l'Assemblée générale» au numéro 62: «Application de la Déclaration sur l'octroi de l'indépendance aux pays et aux peuples coloniaux» (document A/72/10o pour la présente - au moment d'écrire ce texte - session ordinaire de l'Assemblée générale des Nations unies).

3. «La Cour n'a donc pas constaté l'existence de liens juridiques de nature à modifier l'application de la résolution 1514 (XV) quant à la décolonisation du Sahara occidental et en particulier l'application du principe d'autodétermination grâce à l'expression libre et authentique dela volonté des populations du territoire» (Cour internationale de justice, "Sahara occidental, avis consultatif du 16 octobre 1975 ", Recueil des arrêts, avis consultatifs et ordonnances, 1975 , p. $68, \$ 162$ ).

4. Voir, inter alia, A/RES/2229 (XXI), 20 décembre 1966; A/RES/3458 (XXX), 10 décembre 1975; A/RES/38/40, 7 décembre 1983.

5. S/RES/658 (1990), 27 juin 1990; S/RES/690 (1991), 29 avril 1991; S/RES/973 (1995), 13 janvier 1995.

6. Acronyme pour Front Populaire pour la libération de Saguia el Hamra (région nord du Sahara occidental) e Rio de Oro (région sud du Sahara occidental).

7. S/RES/658 (1990), 27 juin 1990; S/RES/690 (1991), 29 avril 1991.

8. Annexes I, II et III du rapport du secrétaire général, S/1997/742, 24 septembre 1997.

9. S/1999/483/Add.1, 13 mai 1999.

10. S/RES/1133 (1997), 20 octobre 1997 et S/RES/1238 (1999), 14 mai 1999.

11. S/RES/690 (1991), 29 avril 1991.

12. $\mathrm{S} / 2000 / 131,23$ février $2000, \$ 6$

13. $\mathrm{S} / 2004 / 325,23$ avril 2004, p. 12.

14. Sur ce sujet, voir C. Ruiz Miguel, «La responsabilité internationale et les droits de l'homme: le cas du Sahara occidental», Cahiers de la recherche sur les droits fondamentaux, $\mathrm{n}^{\circ}$ 11, 2013, p. 105-130.

15. Le texte du plan Baker (officiellement Plan de paix pour l'autodétermination du peuple du Sahara occidental) se trouve en annexe au rapport du secrétaire général au Conseil de sécurité, S/2003/565, 23 mai 2003, annexe II, p. 15-19. 
deux questions: la question des droits de l'homme ${ }^{16}$ et les ressources naturelles du territoire ${ }^{17}$.

L'introduction du vocabulaire des droits de l'homme dans les résolutions du Conseil de sécurité sur le Sahara occidental ne se fit pas de manière aisée. La première référence, indirecte, à la question se trouve dans une résolution de 2010 qui, sous influence autrichienne, évoque la "dimension humaine» du conflit ${ }^{18}$. En effet, la «dimension humaine» est la formule employée par l'Organisation pour la coopération et la sécurité en Europe (OSCE) pour traiter des droits de l'homme ${ }^{19}$, dont le siège se trouve à Vienne... Mais ce n'est que l'année suivante que l'on consacre l'expression des «droits de l'homme» dans le vocabulaire des résolutions du Conseil de sécurité sur le Sahara occidental. C'est en 2011 que le Conseil de sécurité souligne «qu'il importe d'améliorer la situation des droits de l'homme au Sahara occidental ${ }^{20}$, mais sans établir un système international de protection à cet égard. Cette formule fut encore reprise en $2 \mathbf{O O 1 2}^{21}$. Il faudra toutefois attendre 2013 pour que les États-Unis dans leur projet de résolution sur la question du Sahara occidental essaient d'introduire un mécanisme international de suivi, mais la farouche opposition du Maroc, soutenu par la France et l'Espagne, fit échouer cette tentative. En échange, la résolution évoque l' "interaction en cours du Maroc avec les Procédures spéciales du Conseil des droits de l'homme de l'Organisation des Nations Unies ${ }^{22}$. Cette interaction commence en 2010 (avec la mission au Maroc du groupe de travail sur les disparitions forcées ou involontaires ${ }^{23}$ ), continue en 2011 (avec l'experte indépendante dans le domaine des droits culturels ${ }^{24}$ ), en 2012 (avec le groupe de travail sur l'élimination de la discrimination à l'égard des femmes dans la législation et dans la pratique ${ }^{25}$, le rapporteur spécial sur la torture et autres peines ou traitements cruels, inhumains ou dégradants ${ }^{26}$ ) et en 2013 (avec le groupe de travail sur la détention arbitraire ${ }^{27}$, la rapporteuse spéciale sur la traite des êtres humains ${ }^{28}$ ).
Mais après le succès diplomatique franco-marocain au niveau du Conseil de sécurité et la résolution qui loua les avantages des Procédures spéciales du Conseil des droits de l'homme, en 2014, le Maroc n'avait encore reçu la visite d'aucun rapporteur spécial ou groupe de travail. Ce n'est qu'en janvier 2015 que sera autorisée la visite au Sahara occidental de l'experte indépendante sur les droits de l'homme et la solidarité internationale ${ }^{29}$. Mais le Maroc a refusé d'autoriser le suivi des observations déjà formulées par certains rapporteurs spéciaux, notamment celui sur la torture. Les limites de cette formule sont notoires, et le secrétaire général considère qu'une

[...] surveillance indépendante, impartiale, globale et soutenue de la situation des droits de l'homme est nécessaire pour veiller à assurer la protection de tous les habitants du Sahara occidental ${ }^{30}$.

\section{La position du Parlement européen sur le statut international du Sahara occidental et les droits de l'homme}

La Communauté européenne, avant sa transformation en Union européenne, s'était saisie de la question du Sahara occidental. C'est le Parlement européen (PE) qui s'est prononcé sur le statut du territoire, mais aussi sur la question des droits de l'homme.

\section{A. Le statut du Sahara occidental dans les résolutions du Parlement européen ${ }^{31}$}

Le PE s'est prononcé sur le statut du territoire tout en faisant sienne la doctrine des Nations unies. Selon le PE, le Sahara occidental est un «territoire non autonome», objet d'un «processus de décolonisation» qui n'est pas encore parachevé ${ }^{32}$. Le conflit du Sahara occidental est donc un

16. Le $\$ 13$ du plan Baker prévoyait que « [1] es lois, règlements et autres textes adoptés par l’Autorité du Sahara occidental doivent tous être conformes aux normes internationales relatives aux droits de l'homme [...]» (S/2003/565, 23 mai 2003, annexe II, p. 17)

17. Le $₫ 8$.a du plan Baker attribuait « compétence exclusive» à l’Autorité du Sahara occidental, pendant la période transitoire préalable au référendum sur la "pêche» et les «mines» (S/2003/565, 23 mai 2003, annexe II, p. 16).

18. S/RES/1920 (2010), 30 avril 2010, p. 1.

19. Voir V.-Y. Ghebali, Le rôle de l'OSCE en Eurasie, du sommet de Lisbonne au Conseil ministériel de Maastricht (1996-2003), Bruxelles, Bruylant, 2014 , p. 2114 sq.

20. S/RES/1979 (2011), 27 avril 2011, p. 2

21. S/RES/2044 (2012), 24 avril 2012, p. 2

22. S/RES/2099 (2013), 25 avril 2013, p. 2

23. A/HRC/13/31/Add.1, 9 février 2010 .

24. A/HRC/20/26/Add.2, 2 mai 2012

25. A/HRC/20/28/Add.1, 19 juin 2012.

26. A/HRC/22/53/Add.2, 30 avril 2013

27. A/HRC/WGAD/2013/19, 14 janvier 2014; A/HRC/27/48/Add.5, 4 août 2014

28. A/HRC/26/37/Add.3, $1^{\text {er }}$ avril 2014

29. A/HRC/32/43/Add.1, 27 avril 2016.

30. S/2018/277, 29 mars 2018, $\$ 88$; voir aussi S/2017/307, 10 avril 2017, $\$ 92$

31. Voir C. Ruiz Miguel, «La Unión Europea y el Sahara Occidental: (verdaderos) principios y (falsos) intereses», in El derecho a la libre determinación del pueblo del Sahara Occidental: del "ius cogens" al "ius abutendi", F. Palacios Romeo (dir.), Cizur Menor, Thomson-Aranzadi, 2014, p. 161-201; J. D. Torrejón Rodríguez, La Unión Europea y la cuestión del Sahara Occidental: la posición del Parlamento Europeo, Madrid, Reus, 2014; The European Approach towards Western Sahara, M. Balboni, G. Laschi (dir.), Bruxelles, P. Lang, 2017.

32. Résolutions du 27 octobre 2005 et du 25 novembre 2010, Journal officiel de l'Union européenne, 9 novembre 2006, C 272E, p. 582-583; Journal officiel de l'Union européenne, 3 avril 2012, C 99E, p. 87-88. 
"problème de décolonisation ${ }^{33}$ qui oppose deux parties, qui sont le Maroc et le Front Polisario ${ }^{34}$. Le PE reconnaît que la solution de ce conflit nécessite que le "peuple du Sahara occidental» exerce son «droit inaliénable» à «l'autodétermination » et à "l'indépendance » ${ }^{35}$ à travers un référendum ${ }^{36}$. Ce référendum doit être «libre ${ }^{37}$. L'UE ne considère pas que le Sahara occidental appartient au Maroc et qualifie les territoires du Sahara occidental sous contrôle marocain de «territoires occupés $»^{38}$ et recommande

[...] que le territoire du Sahara occidental soit placé provisoirement sous le contrôle des Nations unies et de leur force de paix (MINURSO), en attendant la préparation et la tenue d'un référendum qui permettra au peuple sahraoui de se prononcer sur l'avenir de son pays ${ }^{39}$.

Le $\mathrm{PE}$ a fait référence au statut de l'Espagne dans sa première résolution sur le Sahara occidental, approuvée en 1981 alors que l'Espagne n'avait pas encore adhéré aux Communautés européennes. Alors que les versions française, italienne ou allemande, au niveau du passage faisant état du retrait de l'Espagne, laissent entendre qu'elle est encore la "puissance administrante ${ }^{40}$, le texte anglais semble différent ${ }^{41}$.

\section{B. Les droits de l'homme au Sahara occidental et le Parlement européen}

La première résolution du PE sur le Sahara occidental date du 12 mars $1981^{42}$ mais elle ne fait pas référence aux droits de l'homme. Cependant, à partir de sa deuxième résolution sur la question, le $\mathrm{PE}$ a pris en compte la situation des droits de l'homme au Sahara occidental dans les résolutions approuvées dans les années qui ont suivi ${ }^{43}$, toutes adoptées avant que le Conseil de sécurité n'approuve le Plan de règlement pour le Sahara occidental. Au moment de la publication du Plan, le PE a approuvé une résolution en soutien dudit Plan ${ }^{44}$, mais a réitéré peu après ses demandes de respect des droits de l'homme dans différentes résolutions (12 septembre $1991^{45}, 29$ octobre $1992^{46}, 27$ mai $1993^{47}$, 10 février $1994^{48}$, 16 mars $1995^{49}, 13$ juillet $1995^{50}$ ).

Le 26 février 1996, la Communauté européenne et le Maroc ont signé un accord d'association, entré en vigueur en $2000^{51}$. À partir de ce moment-là, les résolutions du PE deviennent moins exigeantes concernant le respect des droits de l'homme et invitent plutôt le Maroc à un «dialogue large et permanent visant à promouvoir le respect des droits de l'homme», comme le dit la résolution du 6 juin $1996^{52}$. Dans les années suivantes, les droits de l'homme disparaissent du langage du PE (recommandation du 10 mars $1998^{53}$, résolution du 14 janvier $1999^{54}$ ). La résolution du 16 mars 2000 adopte un langage plus ferme en demandant «la garantie d'une liberté totale d'expression, d'association et de manifestation pour les habitants du Sahara occidental $»^{55}$. Mais plusieurs résolutions approuvées postérieurement sont moins exigeantes à ce sujet: la résolution du

33. Résolutions du 15 mars 1989 et du 12 septembre 1991, Journal officiel de l'Union européenne, 17 avril 1989, C 96, p. 59-61; Journal officiel de l'Union européenne, 14 octobre 1991, C 267, p. 129-130.

34. Résolutions du 15 mars 1989, du 11 octobre 1990, du 18 avril 1991 et du 16 mars 1995, Journal officiel de l'Union européenne, 17 avril 1989, C 96, p. 59-61; Journal officiel de l'Union européenne, 12 novembre 1990, C 284, p. 131-132; Journal officiel de l'Union européenne, 20 mai 1991, C 129, p. 126-127; Journal officiel de l'Union européenne, 10 avril 1995, C 89, p. 159.

35. Résolution du 15 mars 1989, Journal officiel de l'Union européenne, 17 avril 1989, C 96, p. 59-61.

36. Résolutions du 15 mars 1989, du 18 avril 1991, du 16 mars 1995, du 16 mars 2000 et résolution ACP-UE du 20 mars 1997, Journal officiel de l'Union européenne, 17 avril 1989, C 96, p. 59-61; Journal officiel de l'Union européenne, 20 mai 1991, C 129, p. 126-127; Journal officiel de l'Union européenne, 10 avril 1995, C 89, p. 159; Journal officiel de l'Union européenne, 29 décembre 2000, C 377, p. 354-355; Journal officiel de l'Union européenne, 9 octobre 1997, C 308 , p. 52.

37. Résolutions du 18 avril 1991 et du 16 mars 200o, Journal officiel de l'Union européenne, 20 mai 1991, C 129, p. 126-127; Journal officiel de l’Union européenne, 29 décembre $2000, \mathrm{C}_{377}$, p. 354-355.

38. Résolution du 27 mai 1993, Journal officiel de l'Union européenne, 28 juin 1993, C 176, p. 158.

39. Résolution du 18 avril 1991, Journal officiel de l'Union européenne, 20 mai 1991, C 129, p. 126-127.

40. Résolution du 12 mars 1981, Journal officiel de l'Union européenne, 6 avril 1981, C 77, p. 43-45 (version française: «à la suite du retrait de l'Espagne, puissance administrante, du Sahara occidental»; version italienne: «in seguito al ritiro della Spagna, potenza amministrativa, dal Sahara occidentale»; version allemande: «infolge des Rückzugs Spaniens als Verwaltungsmacht der westlichen Sahara»).

41. "Following the end of Spanish administrative authority in Western Sahara».

42. Journal officiel de l'Union européenne, 6 avril 1981, C 77, p. 43-45.

43. Résolutions du 17 décembre 1987, du 15 mars 1989, du 15 février 1990 et du 11 octobre 1990, Journal officiel de l'Union européenne, 18 janvier 1988, C 13, p. 102; Journal officiel de l'Union européenne, 17 avril 1989, C 96, p. 59-61; Journal officiel de l'Union européenne, 19 mars 1990, C 68, p. 143-144; Journal officiel de l'Union européenne, 12 novembre 1990, C 284, p. 131-132.

44. Résolution du 18 avril 1991, Journal officiel de l'Union européenne, 20 mai 1991, C 129, p. 126-127.

45. Résolution du 12 septembre 1991, Journal officiel de l'Union européenne, 14 octobre 1991, C 267, p. 129-130.

46. Résolution du 29 octobre 1992, Journal officiel de l'Union européenne, 23 novembre 1992, C 305, p. 153.

47. Résolution du 27 mai 1993, Journal officiel de l'Union européenne, 28 juin 1993, C 176, p. 158.

48. Résolution du 10 février 1994, Journal officiel de l'Union européenne, 28 février 1994, C 61, p. 177.

49. Résolution du 16 mars 1995, Journal officiel de l'Union européenne, 10 avril 1995, C 89, p. 159.

50. Résolution du 13 juillet 1995, Journal officiel de l'Union européenne, 25 septembre 1995, C 249, p. 159-160.

51. Décision du Conseil et de la Commission du 24 janvier 2000 relative à la conclusion de l'accord euro-méditerranéen établissant une association entre les Communautés européennes et leurs États membres, d'une part, et le Royaume du Maroc, d'autre part, 2000/204/CE, CECA, Journal officiel de l'Union européenne, 18 mars $2000, \mathrm{~L} 70, \mathrm{p} .1$.

52. Résolution du 6 juin 1996, Journal officiel de l'Union européenne, 24 juin 1996, C 181, p. 15-16.

53. Recommandation du 10 mars 1998, Journal officiel de l'Union européenne, 6 avril 1998, C 104, p. 29-30.

54. Résolution du 14 janvier 1999, Journal officiel de l'Union européenne, 14 avril 1999, C 104, p. 112-113.

55. Résolution du 16 mars 2000, Journal officiel de l'Union européenne, 29 décembre 2000, C 377, p. 354-355. 
20 novembre $2003^{56}$ n'évoque pas les droits de l'homme et celle du 14 avril $2005^{57}$ observe avec préoccupation les rapports qui dénoncent les violations des droits de l'homme au Sahara occidental sous contrôle marocain, mais ne «demande» pas au Maroc de mettre fin à ces pratiques.

Un mois après cette dernière résolution, le 20 mai 2005 se déclenche une "intifadha" au Sahara occidental occupé. La position du PE va alors s'infléchir. Dans sa résolution du 27 octobre $2005^{58}$, le PE «demande» le « respect» des droits de l'homme et appelle pour la première fois à «la préservation des ressources naturelles énergétiques du Sahara occidental». Après cette résolution, les relais du Maroc réussiront à faire échouer les tentatives de validation des nouvelles résolutions. Revêt à cet égard une importance particulière le projet de résolution du 16 décembre $2009^{59}$ concernant l'affaire de la déportation de $\mathrm{M}^{\mathrm{me}}$ Aminatou Haïdar (prisonnière politique de longue date et symbole de la résistance pacifique) qui demandait au Maroc

[...] de respecter les droits des défenseurs des droits de l'homme sahraouis et de toutes les personnes sous sa juridiction ainsi que le plein exercice des droits et des libertés fondamentales.

Le projet de résolution va avorter in extremis, car le lendemain le Maroc va accepter le retour au Sahara occidental de $\mathrm{M}^{\text {me }}$ Haïdar. Mais, l'année suivante, le Maroc détruit le campement de protestation pacifique érigé à Akdeim Izik, près d'El Aaïun, le chef-lieu du Sahara occidental. C'est alors que le PE donne son feu vert à sa résolution du 25 novembre $2010^{60}$ qui

[...] exprime sa préoccupation face à la détention et aux allégations de harcèlement des défenseurs sahraouis des droits de l'homme sur le territoire du Sahara occidental [...]

et

[...] demande que les défenseurs des droits de l'homme emprisonnés sur ledit territoire ou au Maroc soient traités dans le respect des normes internationales et qu'ils soient jugés rapidement et de façon juste.

Cette résolution introduit un paragraphe très important où l'on
[...] demande à l'Union européenne d'exiger du Royaume du Maroc qu'il se conforme au droit international en ce qui concerne l'exploitation des ressources naturelles au Sahara occidental.

Il est question de toutes sortes de "ressources naturelles» et pas seulement des ressources «énergétiques». À peine un mois après, le $\mathrm{PE}$, dans une nouvelle résolution datée du 16 décembre $2010^{61}$, durcit le ton et «condamne la détention et le harcèlement dont sont victimes les défenseurs des droits de l'homme sahraouis dans le territoire du Sahara-Occidental contrôlé par le Maroc» et «invite instamment les Nations unies à inclure la surveillance de la situation des droits de l'homme dans le mandat de la mission des Nations unies au Sahara-Occidental (Minurso)».

Plusieurs résolutions du PE approuvées les années suivantes continuent à demander le respect des droits de l'homme au Sahara occidental et à réitérer la demande d'un mécanisme international pour sa protection ${ }^{62}$.

\section{La question du Sahara occidental dans les avis du service juridique du Parlement européen}

Lors de la discussion, au $\mathrm{PE}$, sur la ratification de certains accords internationaux avec le Maroc, plusieurs comités du PE avaient demandé des avis juridiques au service juridique du PE. Ces avis sont confidentiels (donc non publiés officiellement), en application de l'article 4(2) du règlement $(\mathrm{CE})$ relatif à l'accès du public aux documents du PE, du Conseil et de la Commission. Cependant, ils ont été totalement ou partiellement communiqués ${ }^{63}$. Il existe, au moins, quatre avis du service juridique du PE concernant le Sahara occidental, datés de 2006, 2009, 2011 et 2013. Trois $(2006,2009,2013)$ concernent les accords de pêche, et l'autre (2011) concerne l'accord de libéralisation des produits agricoles et de pêche. Mais ni les arrêts de la Cour dans les affaires Polisario et Western Sahara Campaign, ni l'avocat général ne citent ces documents qui sont pourtant tout à fait pertinents. Aucun desdits avis ne se penche sur la question des droits de l'homme.

56. Résolution du 20 novembre 2003, Journal officiel de l'Union européenne, 7 avril 2004, C 87E, p. 500-503.

57. Résolution du 14 avril 2005, Journal officiel de l'Union européenne, 9 février 2006, C 33E, p. 596-597.

58. Résolution du 27 octobre 2005, Journal officiel de l'Union européenne, 9 novembre 2006, C 272E, p. 582-583.

59. Projet de résolution du 16 décembre 2009 (http://www.europarl.europa.eu/sides/getDoc.do?pubRef=-//EP//TEXT+MOTION+P7-RC-20o9$0247+0+\mathrm{DOC}+\mathrm{XML}+\mathrm{Vo} / / \mathrm{FR})$

60. Résolution du 25 novembre 2010, Journal officiel de l'Union européenne, 3 avril 2012, C 99E, p. 87-88.

61. Résolution du 16 décembre 2010, Journal officiel de l'Union européenne, 15 juin 2012, C 169E, p. 81-107.

62. Voir les résolutions du 16 février 2012, du 18 avril 2012, du 13 décembre 2012, du 7 février 2013, du 22 octobre 2013 , du 23 octobre 2013 et du 17 décembre 2015, Journal officiel de l'Union européenne, 30 août 2013, C 249E, p. 41-49; Journal officiel de l'Union européenne, 7 septembre 2013, C 258E, p. 8-36; Journal officiel de l'Union européenne, 23 décembre 2015, C 434, p. 87-111; Journal officiel de l'Union européenne, 22 janvier 2016, C 24, p. 89-97; Journal officiel de l'Union européenne, 10 juin 2016, C 208, p. 2-23; Journal officiel de l'Union européenne, 10 juin 2016, C 208, p. 119-136; Journal officiel de l'Union européenne, 24 novembre 2017, C 399, p. 151-175.

63. Règlement (CE) $n^{\circ}$ 1049/2001 du Parlement européen et du Conseil du 30 mai 2001 relatif à l'accès du public aux documents du Parlement européen, du Conseil et de la Commission, article 4(2): «Les institutions refusent l'accès à un document dans le cas où sa divulgation porterait atteinte à la protection: / - des intérêts commerciaux d'une personne physique ou morale déterminée, y compris en ce qui concerne la propriété intellectuelle, / - des procédures juridictionnelles et des avis juridiques, / - des objectifs des activités d'inspection, d'enquête et d'audit, /à moins qu'un intérêt public supérieur ne justifie la divulgation du document visé». 


\section{A. L'avis de 2006}

Le premier (à notre connaissance) avis du PE concernant le Sahara occidental est celui du 20 février $2006^{64}$. Il fut signé par Ricardo Passos et Gabriele Mazzini, ainsi que par Gregorio Garzón Clariana en tant que «Jurisconsult». Cet avis fut demandé par les coordinateurs de la Commission de développement du PE, dans une lettre du 25 janvier 2006. Il se penche sur la compatibilité des accords de pêche avec le droit international sans faire mention des droits de l'homme à cet égard.

Dans son avis, le service juridique rappelle que le Sahara occidental est listé depuis 1963 comme « territoire non autonome» par les Nations unies ${ }^{65}$. Pour le service juridique du PE, l’Espagne est encore considérée comme la «puissance administrante» du territoire même si après 1976 elle n'a pas rempli ses obligations d'information découlant de l'article 73.e de la Charte des Nations unies ${ }^{66}$. Le Maroc, quant à lui, n'est ni puissance «souveraine», ni «administrante» de iure du territoire, et sa présence est seulement factice après l'invasion du territoire qui est donc occupé ${ }^{67}$.

Une fois le statut du territoire déterminé, le service juridique ajoute que, d'après le droit communautaire, les accords internationaux conclus par l'UE doivent respecter les règles du droit international ${ }^{68}$. Le service juridique du PE précise que plusieurs résolutions de l'Assemblée générale des Nations unies déclarent que les activités économiques dans les territoires non autonomes doivent se dérouler en collaboration et au bénéfice des populations autochtones desdits territoires. Cet avis rappelle l'avis du sous-secrétaire général des Nations unies et conseiller juridique de l'organisation qui dans son avis sur le Sahara occidental avait affirmé que les activités d'exploration et d'exploitation dans les territoires non autonomes violent le droit international s'ils méprisent les intérêts et les vœux du peuple du territoire non autonome ${ }^{69}$.

La conclusion du service juridique est que l'accord de pêche de 2006 entre le Maroc et l'UE ne prévoit pas l'inclusion des eaux du Sahara occidental, mais n'exclut pas la possibilité que le Maroc émette des licences de pêche pour opérer dans ces eaux. Pour évaluer la compatibilité de l'accord avec le droit international, il faut savoir comment celui-ci sera mis en œuvre par le Maroc et comment le Maroc en fera profiter les populations locales $^{70}$. Si la mise en œuvre de l'accord soulève des «difficultés» (on sousentend de type juridique) et si le Maroc méconnaît «ses obligations vis-à-vis du droit international envers le peuple du Sahara occidental», l'UE peut éventuellement invoquer la procédure établie dans l'accord pour suspendre son application $^{71}$.

Le service juridique du PE laissait la porte ouverte à une possible extension de l'accord UE-Maroc au Sahara occidental si le Maroc remplissait ses «obligations» découlant du «droit international» envers le "peuple du Sahara occidental». Même si ces obligations n'étaient pas explicitées, le service juridique laissait entendre la possibilité d'exiger du Maroc (puissance «de facto» sur le Sahara occidental) les obligations imposées aux puissances administrantes d'un territoire non autonome.

\section{B. L'avis de 2009}

Le deuxième avis est également requis par la Commission de développement du PE (en l'espèce, par son président, M. Borrell Fontelles), dans une lettre datée du 6 mai 2009. L'avis fut rendu le 13 juillet 2009 et fut signé par Johann $\mathrm{Schoo}^{72}$. Ce nouvel avis essaie de répondre à deux questions: la première est celle de la conséquence de la déclaration par la République arabe sahraoui démocratique (RASD) de sa zone économique exclusive; la seconde est celle des conséquences de la constatation, après l'entrée en vigueur de l'accord de pêche, du fait que les bateaux européens avaient pêché dans les eaux du Sahara occidental.

Le contexte de la première question est particulier : le 21 janvier 2009, la RASD avait approuvé sa loi 03/2009 établissant les zones maritimes de la République ${ }^{73}$. Le président de la Commission de développement demandait au service juridique du PE quelles étaient les conséquences pour l'accord UE-Maroc de cette déclaration. La réponse

64. Avis juridique du service juridique du PE, «Proposal for a Council Regulation on the Conclusion of the Fisheries Partnership Agreement between the European Community and the Kingdom of Morocco - Compatibility with the Principles of International Law», 20 février 2006 , SJ-0085/o6 - D(2006)7352, en ligne: http://www.usc.es/export9/sites/webinstitucional/gl/institutos/ceso/descargas/EU-Parliament-Legalopinion_FPA_2o-February-2006.pdf.

65. Ibid., $\$ 9$ et 37. .

66. Ibid., $\$ 11$ et $37 . b$.

67. Ibid., $\$ 4$ : «After Spain withdrew in 1976, Western Sahara remained de facto under the control of Morocco and Mauritania, whose troops invaded the territory from North and South respectively. Due to pressures by the Polisario Front, in 1979 Mauritania abandoned the occupied regions to which Morocco extended its control, thus becoming the only administrator of Western Sahara".

68. Ibid., $\$ 38$.

69. Ibid., $\$ 39$ et 40

70. Ibid., $\$ 43$

71. Ibid., $\$ 44$; nous traduisons.

72. Avis juridique du service juridique du PE, 13 juillet 2009, SJ-0269/09. Seules les conclusions de l'avis ont fuité. Le texte desdites conclusions peut être consulté en ligne: http://www.usc.es/export9/sites/webinstitucional/gl/institutos/ceso/descargas/EU-Parliament-Legal-opinion_FPA_13July-2009.pdf.

73. Le texte, en anglais, de cette loi peut être consulté en ligne: http://www.usc.es/exportg/sites/webinstitucional/gl/institutos/ceso/descargas/ SADR_Maritime-Law.pdf. La carte maritime des zones établies par cette loi est consultable en ligne: http://www.usc.es/export9/sites/ webinstitucional/gl/institutos/ceso/descargas/Map-SADR-maritime-2010.pdf. 
du service juridique était que ladite déclaration ne produisait aucune conséquence pour trois raisons: en premier lieu parce que pour ledit service la RASD ne jouit pas des caractéristiques d'un État; en deuxième lieu parce que la RASD ne peut pas signer la Convention des Nations unies sur le droit de la mer (UNCLOS); et finalement parce que même si la RASD contrôle seulement une partie du territoire du Sahara occidental, le territoire dans son ensemble est considéré comme un "territoire non autonome» au sens de l'article 73 de la Charte des Nations unies.

La seconde question concerne les captures effectuées par les bateaux de l'UE dans les eaux du Sahara occidental. Si l'avis de 2006 ne répondait pas à la question de la légalité des captures dans les eaux du Sahara occidental parce qu'il n'y avait pas encore de faits pour prouver cette éventualité, l'avis de 2009 reprend la question une fois constaté que les bateaux de l'UE avaient effectivement profité de l'accord UE-Maroc pour pêcher dans les eaux du Sahara occidental. Selon le service juridique du PE, les dotations financières de l'UE sont dépensées pour améliorer les ports du Sahara occidental, ce qui ne signifie pas que le peuple du Sahara occidental en bénéficie, car aucune preuve ne permet de soutenir que ladite réforme des ports bénéficie aux Sahraouis. Le service juridique demande à l'UE de parvenir à un accord amiable avec le Maroc pour vérifier que le peuple sahraoui bénéficie effectivement de l'argent transféré par l'UE. Si ceci s'avère impossible, l'UE peut suspendre l'accord ${ }^{74}$. Si l'UE constate que les accords de pêche sont mis en œuvre sans respecter les principes du droit international sur les droits du peuple sahraoui sur ses ressources naturelles, principes qui sont contraignants pour la Communauté européenne, la CE doit demander des licences de pêche uniquement pour les eaux du Maroc ${ }^{75}$.

\section{L'avis de 2011}

Le troisième avis, daté du 21 janvier 2011, et signé par Ricardo Passos ${ }^{76}$, fut demandé par le président de la Commission du commerce international, le député Vital Moreira, dans une lettre du 11 novembre 2010. Trois questions sont posées au service juridique:

- Est-ce que l'accord de libéralisation réciproque des produits agricoles et halieutiques entre l'UE et le Maroc s'applique aussi au Sahara occidental?;

- Si tel est le cas, est-ce compatible avec les obligations de droit international applicables à l'UE et qui qualifient le Sahara occidental de «territoire non autonome»?;

- Comment doit s'appliquer au Sahara occidental la référence aux « indications géographiques» des produits?
En premier lieu, les conclusions du service juridique n'apportent pas d'éclaircissements quant à la question de savoir si le «texte» de l'accord prévoit son application au Sahara occidental et le service juridique affirme qu'il ne dispose pas de preuves attestant de son application.

À la deuxième question, le service juridique répond affirmativement. À cet égard l'avis de 2011 se distingue de celui de 2006. Cet avis qualifie le Maroc de «puissance administrante de facto » et considère que ne sont pas interdites pour une telle puissance les activités économiques dans un territoire non autonome sur lequel, il faut le rappeler, le Maroc n'est pas «souverain» ni «puissance administrante de iure». Pour le service juridique, une puissance occupante (dans les termes de l'avis, "puissance administrante de facto») jouit des mêmes compétences que les puissances administrantes de iure si elle développe ses activités en accord avec les vœux et les intérêts du peuple des territoires du Sahara occidental, qui doivent bénéficier du développement socio-économique desdits territoires. Le service juridique considère que l'élimination des droits de douane au Sahara occidental peut être considérée comme une contribution au développement socio-économique au sens de l'article 73.a de la Charte des Nations unies, qui est une des obligations imposées aux puissances administrantes des territoires non autonomes. Cependant, le service juridique explique que ces considérations in abstracto doivent être clarifiées en l'espèce pour savoir si les vœux et intérêts du peuple du Sahara occidental sont respectés.

À la troisième question, le service juridique répond que les indications géographiques des produits originaires du Sahara occidental contribueraient à la sauvegarde des cultures traditionnelles, tout comme à la diversité géographique et aux méthodes de production du territoire.

$\mathrm{Si}$, dans son premier avis de 2006 , le service juridique du PE ouvrait la porte à une éventuelle extension de l'accord UE-Maroc au Sahara occidental si le Maroc remplissait ses «obligations » découlant du «droit international» envers le «peuple du Sahara occidental», cet avis de 2011 explicite ces obligations : le peuple du Sahara occidental doit manifester sa volonté de voir appliquer l'accord dans son territoire. Cet accord doit par ailleurs être mis en œuvre dans l'intérêt du peuple du Sahara occidental et lui bénéficier.

\section{L'avis de 2013}

Le quatrième avis du service juridique, daté du 4 novembre 2013 et signé par Daniel Warin et Mihkel Allik, ainsi que par Ricardo Passos, fut demandé par le président de la

74. Ibid., $\$ 8$.

75. Ibid., $\$ 9$.

76. Avis juridique du service juridique du PE, «Agreement between the European Union and the Kingdom of Morocco Concerning Reciprocal Liberalisation Measures on Agricultural Products, Processed Agricultural Products, Fish and Fishery Products», 21 janvier 2111, SJ-o699/10 - RP/ MA/DG/al - D(2010)58129, en ligne : http://www.usc.es/export9/sites/webinstitucional/gl/institutos/ceso/descargas/EU_Parliament-legalopinion_Agric_21-janvier-2011.pdf. 
Commission de la pêche du PE, Gabriel Mato Adrover dans une lettre du 8 octobre $2013^{77}$.

Trois questions sont posées. La première est de savoir si le Maroc avait déclaré les eaux du Sahara occidental comme étant comprises dans sa zone économique exclusive (ZEE). La deuxième est de déterminer si le Maroc avait une base légale pour faire une telle déclaration. Et la troisième est d'identifier la base légale, afin que l'UE fasse des virements financiers pour pêcher dans les eaux « internationales».

La réponse à la première question est claire. Au moment de l'émission de l'avis, le Maroc n'avait pas fait de déclaration de sa ZEE comprenant le Sahara occidental. En fait, lorsqu'après l'invasion du Sahara occidental le Maroc amenda en 1980 sa législation sur la $\mathrm{ZEE}^{78}$, la teneur de la loi resta la même qu'avant l'occupation du Sahara occidental ${ }^{79}$. La loi marocaine n'avait donc pas à ce moment inclus les eaux du Sahara occidental dans la ZEE marocaine.

Pour la deuxième question, le service juridique du $P E$ reprend sa doctrine, seulement suggérée dans son avis de 2006, mais déjà établie explicitement dans son avis de 2009, à savoir que les règles de la Charte des Nations unies pour les «puissances administrantes» (sous-entendu "puissances de iure») sont aussi applicables aux "puissances administrantes de facto». Pour le service juridique du PE, le Maroc peut donc exploiter les eaux de ce qui correspond à une éventuelle ZEE du Sahara occidental lorsqu'il respecte les "vœux» et «intérêts» du "peuple du Sahara occidental ${ }^{80}$.

La troisième question précise qu'une fois constaté que dans le cadre des accords UE-Maroc les bateaux européens pêchent dans les eaux du Sahara occidental, il faut que le Comité de pêche du PE contrôle la manière dont les autorités marocaines en font bénéficier le peuple du Sahara occidental ${ }^{81}$.

En conclusion, le quatrième avis du service juridique du PE, tout en admettant que les eaux du Sahara occidental ne sont pas reconnues comme "marocaines", non seulement au niveau du droit international mais aussi au niveau de la loi marocaine, considère que le statut juridique des «puissances administrantes » peut être élargi aux «puissances administrantes de facto », euphémisme pour les «puissances occupantes».

\section{Statut juridique du Sahara occidental et droits de l'homme dans la jurisprudence de la CJUE}

L'argument fondé sur les droits de l'homme ne fut pas pris en considération par le service juridique du PE pour l'examen de la question du Sahara occidental. En fait, les questions posées au service juridique ignoraient ellesmêmes la question. Mais les droits de l'homme deviendront l'un des arguments visant à contester devant la CJUE la validité des accords UE-Maroc. Cependant, l'argument décisif pour trancher ces affaires sera finalement celui du statut juridique international du territoire.

\section{A. L'affaire dite Polisario (T-512/12 et C-104/16 P)}

\section{La demande du Front Polisario}

L'affaire dite Polisario commence avec une requête introduite par le Front Polisario le 19 novembre 2012. Cette requête revêt une importance particulière parce qu'il s'agit de la première fois (à notre connaissance) dans son histoire que le Front Polisario engage une procédure devant une cour au sujet de l'application du droit international sur le Sahara occidental. Après ce recours (affaire T-512/12), le Front Polisario a introduit une nouvelle requête auprès de la CJUE (affaire T-180/14) et a agi devant les Cours d'Afrique du Sud et du Panama.

Le recours conteste la décision 2012/497/UE du Conseil du 8 mars $2012^{82}$, ainsi que le règlement d'exécution (UE) $\mathrm{n}^{\circ}$ 812/2012 de la Commission du 12 septembre $2012^{83}$. La représentation du Front Polisario fonde son recours sur cinq arguments. Un des arguments est lié aux droits fondamentaux et trois des autres arguments sont axés sur le statut international du territoire ${ }^{84}$.

77. Avis juridique du service juridique du PE, «Protocol between the European Union and the Kingdom of Morocco Setting out the Fishing Opportunities and Financial Contribution Provided for in the Fisheries Partnership Agreement in Force between the Two Parties ", 4 novembre 2013, 2013/o315 (NLE), SJ-o665/13 - MA/gr - D(2013)50041, en ligne: http://www.usc.es/export9/sites/webinstitucional/gl/institutos/ceso/descargas/ EU-Parliament-legal-opinion_FPA_04-nov-2013.pdf.

78. Loi 1-81 du 18 décembre 1980, promulguée par dahir n 1-81-179 du 8 avril 1981, établissant une zone économique exclusive de 200 miles nautiques hors les côtes du Maroc, en ligne: http://www.un.org/depts/los/LEGISLATIONANDTREATIES/PDFFILES/MAR_1981_Act.pdf.

79. Avis juridique du service juridique du PE du 4 novembre 2013, \$9.

80. Ibid., $\$ 18$.

81. Ibid., $\$ 31-32$.

82. Décision 2012/497/UE du Conseil du 8 mars 2012 concernant la conclusion de l'accord sous forme d'échange de lettres entre l'Union européenne et le Royaume du Maroc relatif aux mesures de libéralisation réciproques en matière de produits agricoles, de produits agricoles transformés, de poissons et de produits de la pêche, au remplacement des protocoles $n^{\circ} 1,2$ et 3 et de leurs annexes et aux modifications de l'accord euroméditerranéen établissant une association entre les Communautés européennes et leurs États membres, d'une part, et le Royaume du Maroc, d'autre part, Journal officiel de l'Union européenne, 7 septembre 2012, L 241, p. 2.

83. Règlement d'exécution (UE) $n^{\circ} 812 / 2012$ de la Commission du 12 septembre 2012 portant modification du règlement $(\mathrm{CE}) \mathrm{n}^{\circ} 747 / 2001 \mathrm{du}$ Conseil en ce qui concerne les contingents tarifaires de l'Union pour certains produits agricoles et produits agricoles transformés originaires du Maroc, Journal officiel de l'Union européenne, 13 septembre 2012, L 247, p. 7.

84. CJUE, recours introduit le 19 novembre 2012, Front Polisario c. Conseil, affaire T-512/12, Journal officiel de l'Union européenne, 23 février 2013 , C 55, p. 14-15. 
D'après le deuxième argument, la conclusion par l'UE de l'accord avec le Maroc serait illégale parce qu'elle signifie une

[...] violation des droits fondamentaux protégés par l'article 67 TFUE [traité sur le fonctionnement de l'Union européenne], l'article 6 TUE et les principes posés par la jurisprudence en bafouant le droit à l'autodétermination du peuple sahraoui et en encourageant la politique d'annexion conduite par le Royaume du Maroc, puissance occupante selon la partie requérante. La partie requérante fait en outre valoir une violation du principe de cohérence prévu à l'article 7 TFUE par le non-respect du principe de souveraineté, ainsi qu'une violation des valeurs fondant l'Union européenne et des principes présidant son action extérieure en contradiction avec les articles 2 TUE, 3 , paragraphe 5, TUE, 21 TUE et 205 TFUE $^{85}$.

Trois autres arguments tournent autour du statut international du territoire. Ainsi, d'après le troisième argument, les décisions sont contestées parce qu'elles entraînent une

[...] violation des accords internationaux conclus par l'Union européenne, et notamment de l'accord d'association conclu entre l'Union européenne et le Royaume du Maroc, ainsi que de la Convention des Nations unies sur le droit de la mer ${ }^{86}$.

Ensuite, le quatrième argument précise qu'existe une

[...] violation de plusieurs normes de droit international, dont le droit à l'autodétermination, l'effet relatif des traités et les dispositions essentielles du droit international humanitaire ${ }^{87}$.

Finalement, le cinquième argument précise que «l'illicéité du comportement de l'Union européenne en vertu du droit international entraîne l'illégalité de ces actes ${ }^{88}$.

\section{L'arrêt de la $8^{e}$ chambre de la CJUE du 10 décembre 2015: les droits de l'homme comme critère décisif}

Trois ans après l'introduction de la requête, la $8^{\mathrm{e}}$ chambre de la CJUE ${ }^{89}$ prononça sa décision, le 10 décembre $2015^{\circ \circ}$. L'arrêt commence par un excellent exposé, documenté et rigoureux, du statut juridique du Sahara occidental ${ }^{91}$, après quoi il renvoie aux circonstances de l'accord contesté. Plusieurs questions ont été discutées dans le processus (l'audience publique eut lieu le 15 juin 2015) tranchées par la Cour.
En premier lieu, le Front Polisario possède la personnalité juridique pour ester en justice. Le premier aspect discuté était de savoir si le Front Polisario était une "personne morale» au sens de l'article 263 du traité sur le fonctionnement de l'Union européenne (TFUE). La réponse de la Cour est claire et positive. Le paragraphe 60 de l'arrêt se lit comme suit:

6o. Compte tenu de ces circonstances fort particulières, il convient de conclure que le Front Polisario doit être considéré comme une "personne morale", au sens de l'article 263, quatrième alinéa, TFUE, et qu'il peut introduire un recours en annulation devant le juge de l'Union, quand bien même il ne disposerait pas de la personnalité juridique selon le droit d'un État membre ou d'un État tiers. En effet, ainsi que cela a été relevé ci-dessus, il ne saurait disposer d'une telle personnalité que conformément au droit du Sahara occidental qui n'est toutefois, à l'heure actuelle, pas un État reconnu par l'Union et ses États membres et ne dispose pas de son propre droit ${ }^{92}$.

En deuxième lieu, même si le Maroc et l'UE peuvent avoir une idée différente du statut du territoire du Sahara occidental, la Cour conclut que les accords entre l'UE et le Maroc sont applicables au Sahara occidental:

103. Dans ces conditions, il convient de conclure que l'accord dont la conclusion a été approuvée par la décision attaquée, replacé dans son contexte tel qu'il a été défini ci-dessus, s'applique également au territoire du Sahara occidental ou, plus précisément, à la plus grande partie de ce territoire, contrôlée par le Royaume du Maroc ${ }^{93}$.

En troisième lieu, le Front Polisario est directement et individuellement concerné par les accords de l'UE avec le Maroc qui affectent le Sahara occidental. Même en admettant que le Front Polisario possède une personnalité juridique, la question suivante était de savoir s'il pouvait contester cet accord. L'article 263 du TFUE exige que le requérant soit «directement et individuellement» concerné par la règle qu'il conteste. La Cour, encore une fois, répond avec force que le Front Polisario est directement et individuellement concerné par cet accord et que son recours est recevable:

113. Or, les circonstances mentionnées au point 110 cidessus constituent bien une situation de fait qui caractérise le Front Polisario par rapport à toute autre personne et lui confère une qualité particulière. En effet, le Front Polisario est le seul autre interlocuteur qui participe aux négociations menées sous l'égide de l'ONU, entre lui et le Royaume du Maroc, en vue de la détermination du statut international définitif du Sahara occidental.

89. La chambre était composée de M. D. Gratsias (rapporteur), président, $\mathrm{M}^{\mathrm{me}} \mathrm{M}$. Kancheva et M. C. Wetter, juges.

90. CJUE, arrêt du tribunal ( $8^{\mathrm{e}}$ chambre), 10 décembre 2015, Front Polisario $c$. Conseil, affaire T-512/12, en ligne: http://curia.europa.eu/juris/celex. jsf? celex $=62012$ TJ0512\&lang $1=$ fr\&type $=$ TXT\&ancre $=$.

91. Ibid., $\$ 1$ à 16 .

92. Ibid.

93. Ibid. 
114. Il convient donc de conclure que, dès lors que le Front Polisario est directement et individuellement concerné par la décision attaquée, il n'existe de ce point de vue aucun doute quant à la recevabilité du recours, contrairement à ce que font valoir le Conseil et la Commission ${ }^{94}$.

En quatrième lieu, l'exploitation des ressources du Sahara occidental doit profiter aux Sahraouis, mais la vérification des profits de cette exploitation ne doit pas être faite par le Maroc. Il revient au Conseil de l'UE de vérifier ce point:

238. [...] l'exportation vers l'Union de produits en provenance, notamment, du Sahara occidental est facilitée par l'accord en question. En effet, cela fait partie des objectifs dudit accord. Par conséquent, s'il devait s'avérer que le Royaume du Maroc exploitait les ressources du Sahara occidental au détriment de ses habitants, cette exploitation pourrait être indirectement encouragée par la conclusion de l'accord approuvé par la décision attaquée.

239. [...] il suffit de relever que l'accord ne garantit pas davantage une exploitation des ressources naturelles du Sahara occidental profitable à ses habitants.

[...]

246. Les arguments du Conseil, résumés aux points 230 et 236 ci-dessus, montrent au contraire qu'il considère que la question de savoir si l'exploitation des ressources du Sahara occidental se fait ou non au détriment de la population locale ne concerne que les autorités marocaines. Or, pour les motifs exposés aux points 227 à 233 ci-dessus, cette thèse ne saurait être admise.

247. Il en résulte que le Conseil a manqué à son obligation d'examiner, avant l'adoption de la décision attaquée, tous les éléments du cas d'espèce. Par conséquent, il convient de faire droit au recours et d'annuler la décision attaquée en ce qu'elle approuve l'application de l'accord visé par elle au Sahara occidental 95 .

En cinquième lieu, afin de remplir l'obligation de vérifier que l'exploitation des ressources économiques du Sahara occidental ne se fait pas au détriment de la population sahraouie, l'UE doit vérifier la présence ou non de violations des droits de l'homme. La Cour de justice de l'UE pose ici une affirmation très importante, à savoir que, si l'UE autorise l'importation de produits obtenus dans un pays tiers en violation des droits fondamentaux, elle encouragerait indirectement ces violations des droits. Cela est particulièrement important dans une situation telle que celle du Sahara occidental, parce que le tribunal dit que non seulement aucun État ne reconnaît que le Sahara occidental est partie intégrante des frontières marocaines (souveraineté), mais également qu'aucune instance internationale ne lui a donné un mandat pour gérer (administration) le Sahara occidental:

231. [...] si l'Union permet l'exportation vers ses États membres de produits en provenance de cet autre pays qui ont été fabriqués ou obtenus dans des conditions qui ne respectent pas les droits fondamentaux de la population du territoire dont ils proviennent, elle risque d'encourager indirectement de telles violations ou d'en profiter.

232. Cette considération est d'autant plus importante dans le cas d'un territoire, comme le Sahara occidental, qui est administré, dans les faits, par un État tiers, en l'occurrence le Royaume du Maroc, tout en n'étant pas inclus dans les frontières internationalement reconnues de cet État tiers.

233. Il convient également de tenir compte du fait que le Royaume du Maroc ne dispose d'aucun mandat, décerné par l'ONU ou par une autre instance internationale, pour l'administration de ce territoire et qu'il est constant qu'il ne transmet pas à l'ONU de renseignements relatifs à ce territoire, tels que ceux prévus par l'article 73, sous e), de la charte des Nations unies.

[...]

241. Or, compte tenu notamment du fait que la souveraineté du Royaume du Maroc sur le Sahara occidental n'est reconnue ni par l'Union et ses États membres ni, plus généralement, par l'ONU, ainsi que de l'absence de tout mandat international susceptible de justifier la présence marocaine sur ce territoire, le Conseil, dans le cadre de l'examen de tous les éléments pertinents du cas d'espèce en vue de l'exercice de son large pouvoir d'appréciation concernant la conclusion, ou non, d'un accord avec le Royaume du Maroc susceptible de s'appliquer également au Sahara occidental, devait s'assurer lui-même qu'il n'existait pas d'indices d'une exploitation des ressources naturelles du territoire du Sahara occidental sous contrôle marocain susceptible de se faire au détriment de ses habitants et de porter atteinte à leurs droits fondamentaux. Il ne saurait se limiter à considérer qu'il incombe au Royaume du Maroc d'assurer qu'aucune exploitation de cette nature n'a lieu ${ }^{96}$.

$\mathrm{Au}$ vu des tous ces arguments, la Cour statua que la décision 2012/497/UE du Conseil, du 8 mars 2012, était annulée en ce qu'elle approuvait l'application dudit accord au Sahara occidental.

En conclusion, l'arrêt de 2015 permet une exploitation des ressources du Sahara occidental même si ce territoire est «tiers», étant donné que le Maroc n’y exerce pas d'autorité au titre de «souveraineté» ni de «puissance administrante». Mais cette exploitation doit se faire en respectant les «intérêts» du peuple sahraoui, exigence déjà établie au niveau des Nations unies ainsi que dans les avis du service juridique du PE. Certes, l'arrêt de 2015 ne retient pas (au moins explicitement) l'obligation de prendre en compte les «vœux» du peuple du Sahara occidental, mais il introduit une exigence nouvelle: que cette exploitation se déroule dans un contexte de respect des droits fondamentaux vérifié par l'UE elle-même. Il restait à clarifier si cette formule visait l'inclusion du droit à l'autodétermination (reconnu internationalement au peuple du Sahara occidental), ou l'obligation de prendre en compte les «vœux» du peuple sahraoui comme l'exigeaient les Nations unies et les avis du service juridique du PE. En tout cas, l'arrêt fut l'objet de critiques en raison de 
son incohérence vis-à-vis des règles du droit international menant inéluctablement à l'interdiction d'un accord visant le Sahara occidental ${ }^{97}$.

\section{Le pourvoi en appel et l'opinion de l'avocat général du 13 septembre 2016}

La défaite du Conseil dans l'affaire qui l'opposa au Front Polisario fut mal reçue. Au lendemain de la publication de l'arrêt, la haute représentante/vice-présidente de la Commission européenne, Federica Mogherini, annonça que

Les institutions européennes sont en train d'examiner attentivement cet arrêt afin de définir les différentes options, notamment les travaux préparatoires en vue de faire appel ${ }^{98}$.

Le pourvoi en appel fut formalisé le 19 février $2016^{99}$. Six arguments du Conseil visaient à casser l'arrêt de la $8^{\mathrm{e}}$ chambre:

- le Conseil estimait que le Tribunal avait commis une erreur de droit en concluant que le requérant «avait qualité pour agir devant la juridiction de l'Union européenne ${ }^{100}$;

- d'après le Conseil, le Tribunal avait commis une erreur de droit en concluant que le requérant était «directement et individuellement concerné par la décision $»^{101}$ annulée;

- le Conseil reprochait au Tribunal d'avoir commis une erreur de droit en fondant l'annulation qu'il avait prononcée sur un moyen qui n'avait pas été invoqué par le requérant et sur lequel le Conseil n'a pas eu la possibilité de se défendre;

- le Conseil faisait grief au Tribunal d'avoir commis une erreur de droit en concluant que le Conseil était tenu d'examiner, avant d'adopter la décision annulée, l'impact possible sur les droits de l'homme de la population du Sahara occidental des activités de production des produits couverts par l'accord conclu par la décision annulée;

- selon le Conseil, le Tribunal aurait commis une erreur de droit en concluant que le Conseil était tenu d'examiner, avant d'adopter la décision annulée, qu'il n'existait pas d'indices d'une exploitation, sous l'accord conclu par ladite décision, des ressources naturelles du territoire du Sahara occidental sous contrôle marocain susceptible de se faire au détriment de ses habitants et de porter atteinte à leurs droits fondamentaux;

- en dernier lieu, le Conseil faisait valoir que le Tribunal avait commis une erreur de droit en opérant une annulation partielle de la décision contestée ayant pour effet de modifier la substance de celle-ci.

Le 13 septembre 2016 furent présentées les conclusions de l'avocat général, Melchior Wathelet ${ }^{102}$. L'avocat général exprime ses appréciations à l'égard des moyens d'appel présentés par le Conseil, et considère que tous les six devraient être rejetés. Dans son examen du cinquième moyen du pourvoi en appel introduit par le Conseil, il est important de souligner que, pour l'avocat général, le droit à l'autodétermination est un des droits fondamentaux que l'UE doit respecter:

259. Par conséquent, avant de conclure des accords internationaux, les institutions de l'Union doivent s'assurer du respect de la liste très restreinte des normes impératives du droit international (ius cogens) et des obligations erga omnes, qui incluent «la mise hors la loi des actes d'agression et du génocide mais aussi des principes et des règles concernant les droits fondamentaux de la personne humaine, y compris la protection contre la pratique de l'esclavage et la discrimination raciale » ainsi que le droit à l'autodétermination ${ }^{103}$.

Mais l'avocat général introduit des considérations additionnelles qui seront d'une grande importance dans l'arrêt définitif de la grande chambre.

La première considération de l'avocat général vise le statut du Sahara occidental tel que déterminé par l'article 73 de la charte des Nations unies et ses conséquences ${ }^{104}$ et conclut que le Sahara occidental possède, en vertu de la Charte des Nations unies,

[...] un statut séparé et distinct de celui de l'État qui l'administre [...] aussi longtemps que le peuple [...] du territoire non autonome n'exerce pas son droit à disposer de luimême conformément à la Charte et, plus particulièrement, à ses buts et principes.

Par conséquent, le champ d'application territorial des accords en cause ne peut couvrir le Sahara occidental.

97. Voir F. Dubuisson, G. Poissonnier, «La Cour de justice de l’Union européenne et la question du Sahara occidental: cachez cette pratique (illégale) que je ne saurais voir", Revue belge de droit international, vol. 49, nº 2, 2016, p. 599-634.

98. Déclaration de la haute représentante/vice-présidente de la Commission européenne Federica Mogherini sur l'arrêt du Tribunal de l'Union européenne, 11 décembre 2015, en ligne: https://eeas.europa.eu/headquarters/headquarters-homepage/550o/declaration-de-la-hrvp-federicamogherini-sur-larret-du-tribunal-de-lunion-europeenne_fr.

99. Journal officiel de l'Union européenne, 29 mars 2016, C 111, p. 17

100. Ibid.

101. Ibid.

102. Conclusions de l'avocat général M. Melchior Wathelet présentées le 13 septembre 2016, affaire C-104/16 P, Conseil de l'Union européenne contre Front populaire pour la libération de la saguia-el-hamra et du rio de oro (Front Polisario), en ligne: http://curia.europa.eu/juris/celex.jsf?celex= 62016CCo104\&lang $1=$ fr\&type $=$ TXT\&ancre $=$

103. Ibid.

104. Notamment la Déclaration relative aux principes du droit international touchant les relations amicales et la coopération entre les États conformément à la charte des Nations unies, approuvée par la résolution 2625 (XXV), du 24 octobre 1970, de l'Assemblée générale de l'ONU. 
Le Sahara occidental ne peut faire partie du territoire du Royaume du Maroc au sens de l'article 94 de l'accord d'association et, par conséquent, les accords d'association et de libéralisation ne lui sont pas applicables ${ }^{105}$.

La seconde considération est que le Sahara occidental jouissant d'un «statut séparé et distinct» du Maroc ou de l'Espagne (comme puissance administrante de iure) l'application au Sahara occidental des accords entre l'UE et le Maroc «serait également contraire au principe général de droit international de l'effet relatif des traités (pacta tertiis nec nocent nec prosunt)» qui trouve une expression particulière dans l'article 34 de la Convention de Vienne. Dans cette hypothèse, le recours en annulation du Front Polisario devrait être rejeté comme irrecevable, faute d'un intérêt et d'une qualité pour agir. En effet, si l'accord de libéralisation n'est pas applicable au Sahara occidental, l'annulation de la décision litigieuse ne pourrait lui procurer un quelconque bénéfice ni l'affecter directement et individuellement ${ }^{106}$.

\section{L'arrêt de la grande chambre de la CJUE du 21 décembre 2016}

La grande chambre a tranché définitivement l'affaire dans son arrêt du 21 décembre $2016^{107}$. La teneur de la décision se laisse deviner lorsque l'arrêt commence avec un exposé du cadre juridique de l'affaire mentionnant la Charte des Nations unies et la Convention de Vienne sur le droit des traités, y compris son article 34 («Un traité ne crée ni obligations ni droits pour un État tiers sans son consentement»).

La prémisse fondamentale de l'arrêt de la $8^{\mathrm{e}}$ chambre était que les accords entre l'UE et le Maroc étaient applicables au Sahara occidental ${ }^{108}$. L'avocat général contesta cette affirmation considérant que «les accords d'association et de libéralisation ne lui sont pas applicables ${ }^{109}$. La grande chambre va partager l'avis de l'avocat général:

92. Compte tenu du statut séparé et distinct reconnu au territoire du Sahara occidental, en vertu du principe d'autodétermination, par rapport à celui de tout État, en ce compris le Royaume du Maroc, les termes «territoire du Royaume du Maroc» figurant à l'article 94 de l'accord d'association ne peuvent, comme le soutient la Commission et comme M. l'avocat général l'a en substance relevé aux points 71 et 75 de ses conclusions, être interprétés de sorte que le Sahara occidental soit inclus dans le champ d'application territorial de cet accord.

[...]

10o. Enfin, il importe de souligner que, en vertu du principe de droit international général de l'effet relatif des traités, dont la règle figurant à l'article 34 de la convention de Vienne constitue une expression particulière, les traités ne doivent ni nuire ni profiter à des sujets tiers sans leur consentement (voir arrêt du 25 février 2010, Brita, C-386/o8, EU:C:2010:91, points 44 et 52).

[...]

103. Or, contrairement à ce qu'a estimé le Tribunal, le principe de l'effet relatif des traités devait être pris en considération dans le cadre d'une telle interprétation, dès lors qu'une application au Sahara occidental de l'accord d'association, conclu entre l'Union et le Royaume du Maroc, aurait conduit à ce que cet accord affecte un «tiers».

104. En effet, il convient de rappeler que, dans son avis consultatif sur le Sahara occidental, auquel le Tribunal s'est lui-même référé au point 8 de l'arrêt attaqué, la Cour internationale de justice a considéré que le Sahara occidental «n'était pas un territoire sans maître (terra nullius) au moment de sa colonisation par 1[e Royaume d]'Espagne», d'une part, et que les éléments et les renseignements portés à sa connaissance "n'établiss[ai]ent l'existence d'aucun lien de souveraineté territoriale» entre ce territoire et le Royaume du Maroc, d'autre part.

105. Plus précisément, à cet égard, la Cour internationale de justice a souligné, dans son avis consultatif sur le Sahara occidental, que la population de ce territoire jouissait, en vertu du droit international général, du droit à l'autodétermination, ainsi que cela est exposé aux points 90 et 91 du présent arrêt, étant entendu que, pour sa part, l'Assemblée générale de l'ONU a, au point 7 de sa résolution $34 / 37$ sur la question du Sahara occidental, citée au point 35 du présent arrêt, recommandé que le Front Polisario, «représentant du peuple du Sahara occidental, participe pleinement à toute recherche d'une solution politique juste, durable et définitive de la question du Sahara occidental», ainsi que le Tribunal l'a indiqué au point 14 de l'arrêt attaqué et que la Commission l'a rappelé devant la Cour.

106. Compte tenu de ces éléments, le peuple du Sahara occidental doit être regardé comme étant un "tiers» au sens du principe de l'effet relatif des traités, ainsi que M. l'avocat général l'a en substance relevé au point 105 de ses conclusions. En tant que tel, ce tiers peut être affecté par la mise en œuvre de l'accord d'association en cas d'inclusion du territoire du Sahara occidental dans le champ d'application dudit accord, sans qu'il soit nécessaire de déterminer si une telle mise en œuvre serait de nature à lui nuire ou au contraire à lui profiter. En effet, il suffit de relever que, dans un cas comme dans l'autre, ladite mise en œuvre doit recevoir le consentement d'un tel tiers. Or, en l'occurrence, l'arrêt attaqué ne fait pas apparaître que le peuple du Sahara occidental ait manifesté un tel consentement.

107. Dans ces conditions, le fait de considérer que le territoire du Sahara occidental relève du champ d'application de l'accord d'association est contraire au principe de droit international de l'effet relatif des traités, lequel est applicable dans les relations entre l'Union et le Royaume du Maroc.

108. Compte tenu des considérations qui précèdent, le Tribunal a commis une erreur de droit en estimant, aux points 101 et 103 de l'arrêt attaqué, que l'Union et le Royaume du Maroc devaient être regardés comme ayant été tacitement d'accord pour interpréter les termes

105. Conclusions de l'avocat général M. Melchior Wathelet présentées le 13 septembre 2016, $\$ 69-82$.

106. Ibid., \$101-115.

107. CJUE, arrêt de la Cour (GC), 21 décembre 2016, Conseil c. Front Polisario, affaire C-104/16 P.

108. CJUE, arrêt du tribunal ( $8^{\mathrm{e}}$ chambre), 10 décembre 2015, Front Polisario c. Conseil, $\$ 103$.

109. Conclusions de l'avocat général M. Melchior Wathelet présentées le 13 septembre $2016, \S 82$. 
«territoire du Royaume du Maroc» figurant à l'article 94 de l'accord d'association en ce sens qu'ils incluaient le territoire du Sahara occidental ${ }^{110}$.

La grande chambre n'a pas besoin d'argumenter sur la base des droits fondamentaux parce qu'elle fonde sa décision sur la base du statut international du Sahara occidental. Lorsque ce territoire jouit d'un statut séparé et distinct des autres États (y compris, bien sûr, le Maroc) avec un droit à l'autodétermination internationalement consacré, il est un «tiers» pour le droit international. En tant que «tiers» le droit des traités n'autorise pas que lui soit applicable une convention signée entre deux autres acteurs internationaux. La Cour, donc, ne prête aucune virtualité à la pseudo-catégorie de "puissance administrante de facto » admise par le service juridique du PE pour valider une application per analogiam du statut de «puissance administrante» au profit du Maroc.

\section{B. L'affaire dite Western Sahara Campaign (C-266/16)}

\section{La question préjudicielle de la High Court of Justice (England \& Wales)}

Une association britannique de soutien au peuple sahraoui (Western Sahara Campaign) avait saisi la Cour contre le ministère britannique de l'Agriculture (Secretary of State for Environment, Food and Rural Affairs) et l'Autorité des impôts et douanes (Commissioners for Her Majesty's Revenue and Customs) pour contester plusieurs agissements officiels britanniques concernant le Sahara occidental ${ }^{111}$. Le 27 avril 2016, la Cour suprême d'Angleterre et du Pays de Galles décide de demander une question préjudicielle ${ }^{112}$, en posant quatre questions à la CJUE:

- si les références au «Maroc» figurant dans l'accord euro-méditerranéen établissant une association entre les Communautés européennes et leurs États membres, d'une part, et le Royaume du Maroc, d'autre $\operatorname{part}^{113}$, renvoient uniquement au territoire souverain du Maroc, tel que reconnu par les Nations unies et l'UE, excluant ainsi que des produits originaires du Sahara occidental soient admis à l'importation dans l'UE en exemption de droits de douane, comme le prévoit l'accord d'association;

- si les produits originaires du Sahara occidental peuvent être admis à l'importation dans l'UE en exemption de droits de douane en vertu de l'accord d'association. La cour anglaise demande si cet accord est valide, compte tenu des dispositions de l'article 3, paragraphe 5, du traité sur l'UE, qui imposent l'obligation de contribuer au respect de tout principe pertinent du droit international et au respect des principes de la Charte des Nations unies et compte tenu de l'accord d'association qui a été conclu au bénéfice du peuple sahraoui, en son nom, conformément à sa volonté, ou en consultation avec ses représentants reconnus;

- si l'accord de partenariat dans le secteur de la pêche entre l'UE et le Royaume du Maroc ${ }^{114}$ (tel qu'approuvé et mis en œuvre par le règlement $n^{\circ} 764 / 2006 \mathrm{du}$ Conseil ${ }^{115}$, le règlement $n^{\circ} 1270 / 2013$ du Conseil ${ }^{116}$ et la décision 2013/785 du Conseil ${ }^{117}$ ) est valide, compte tenu des dispositions de l'article 3, paragraphe 5, du traité sur l'UE, qui imposent l'obligation de contribuer au respect de tout principe pertinent du droit international et au respect des principes de la charte des Nations unies, rappelant que l'accord de partenariat dans le secteur de la pêche a été conclu au bénéfice du peuple sahraoui, en son nom, conformément à sa volonté, ou en consultation avec ses représentants reconnus;

- finalement, la cour anglaise demande si la partie requérante (Western Sahara Campaign) a le droit de contester la validité d'actes de l'Union au motif que l'Union aurait violé le droit international.

\section{L'opinion de l'avocat général du 10 janvier 2018}

L'avocat général va publier ses conclusions le 10 janvier $2018^{118}$. Dans cette affaire, l'avocat général profite de l'occasion pour reformuler ses positions déjà exprimées lors de l'affaire C-104/16 P, mais aussi pour clarifier certains concepts ou expressions.

110. CJUE, arrêt de la Cour (GC), 21 décembre 2016

111. High Court of Justice (England \& Wales), 19 octobre 2015, Western Sahara Campaign UK, $R$ (on the application of) $v$ HM Revenue and Customs, [2015] EWHC 2898 (Admin) / 2015 WL 5949373, affaire $n^{\circ} \mathrm{CO} / 1032 / 2015$ \& 1034/2015.

112. Demande de question préjudicielle présentée par la High Court of Justice (England \& Wales), Queen's Bench Division (Administrative Court) (Royaume-Uni), 13 mai 2016, Western Sahara Campaign UK/Commissioners for Her Majesty's Revenue and Customs, Secretary of State for Environment, Food and Rural Affairs, affaire C-266/16.

113. Accord euro-méditerranéen établissant une association entre les Communautés européennes et leurs États membres, d'une part, et le Royaume du Maroc, d'autre part, Journal officiel de l'Union européenne, 18 mars 2000, L 70, p. 2, approuvé par la décision 2000/204/CE, CECA.

114. Accord de partenariat dans le secteur de la pêche entre la Communauté européenne et le Royaume du Maroc, Journal officiel de l'Union européenne, 29 mai 2006, L 141, p. 4.

115. Règlement (CE) $n^{\circ} 764 / 2006$ du Conseil du 22 mai 2006, Journal officiel de l'Union européenne, 29 mai $2006, \mathrm{~L} 141, \mathrm{p} .1$.

116. Règlement (UE) $n^{\circ}$ 1270/2013 du Conseil du 15 novembre 2013 relatif à la répartition des possibilités de pêche au titre du protocole entre l'Union européenne et le Royaume du Maroc fixant les possibilités de pêche et la contrepartie financière prévues par l'accord de partenariat dans le secteur de la pêche entre l'Union européenne et le Royaume du Maroc, Journal officiel de l'Union européenne, 7 décembre 2013 , L 328, p. 40.

117. Décision 2013/785/UE du Conseil du 16 décembre 2013 relative à la conclusion, au nom de l'Union européenne, du protocole entre l'Union européenne et le Royaume du Maroc fixant les possibilités de pêche et la contrepartie financière prévues par l'accord de partenariat dans le secteur de la pêche entre l'Union européenne et le Royaume du Maroc, Journal officiel de l'Union européenne, 21 décembre 2013 , L 349 , p. 1.

118. Conclusions de l'avocat général M. Melchior Wathelet, 10 janvier 2018, Western Sahara Campaign, affaire C-266/16. 
L'avocat général rappelle que le Sahara occidental est un «tiers» envers le Maroc et l'UE, un tiers qui jouit d'un droit à l'autodétermination, une doctrine déjà établie par la CJUE dans l'affaire Polisario:

143. Par son arrêt du 21 décembre 2016, Conseil/ Front Polisario (C-104/16 P, EU:C:2016:973), la Cour a jugé que l'accord d'association conclu entre l'Union et le Royaume $\mathrm{du}$ Maroc, qui selon son libellé s'applique au «territoire du Royaume du Maroc», n'est pas applicable au territoire du Sahara occidental puisque pareille application serait incompatible avec le droit du peuple de ce territoire à l'autodétermination ainsi qu'avec les articles 29 (application territoriale des traités) et 34 (principe de l'effet relatif des traités selon lequel les traités ne doivent ni nuire ni profiter aux tiers sans leur consentement) de la convention de Vienne sur le droit des traités ${ }^{119}$.

Mais, contrairement à l'affaire antérieure, l'avocat général considère cette fois que les accords (de pêche) qui s'appliquent au Sahara occidental doivent être considérés comme illégaux:

145. Je ne suis pas persuadé par cette argumentation. Si l'application au Sahara occidental d'un accord international conclu avec le Royaume du Maroc dont le champ d'application territorial, n'inclut pas explicitement ce territoire était incompatible avec le droit du peuple de ce territoire à l'autodétermination, un accord international qui, comme l'accord de pêche et le protocole de 2013, est applicable au territoire du Sahara occidental et aux eaux $\mathrm{y}$ adjacentes et autorise une exploitation par l'Union des ressources halieutiques du Sahara occidental le serait a fortiori aussi.

146. Cet argument a fortiori me paraît suffisant pour constater une violation du droit du peuple du Sahara occidental à l'autodétermination. Par souci d'être complet, je voudrais ajouter que les actes contestés ne respectent pas le droit du peuple du Sahara occidental à l'autodétermination en ce qu'ils ne correspondent ni à une poursuite libre de son développement économique ni à une disposition libre de ses richesses et de ses ressources naturelles et que, de toute façon, même s'ils ne violaient pas en eux-mêmes le droit à l'autodétermination, ils ne respecteraient pas l'obligation de l'Union de ne pas reconnaître une situation illicite découlant de la violation du droit du peuple du Sahara occidental à l'autodétermination et de ne pas prêter aide ou assistance au maintien de cette situation ${ }^{120}$.

Pour l'avocat général, le Sahara occidental est victime d'une intégration forcée et illégale dans le Maroc de laquelle découle l'absence d'une libre expression des vœux du peuple du Sahara occidental sur la disposition de ses ressources naturelles:

185. Il résulte de ce qui précède que le Sahara occidental a été intégré au Royaume du Maroc sans que le peuple de ce territoire ait librement exprimé sa volonté à cet égard. L'accord de pêche et le protocole de 2013 étant conclus par le Royaume du Maroc sur la base de l'intégration unilatérale du Sahara occidental à son territoire et de l'affirmation de sa souveraineté sur ce territoire, il est clair que le peuple du Sahara occidental n'a pas librement disposé de ses ressources naturelles, comme l'imposent l'article $1^{\mathrm{er}}$ commun au PIDESC [Pacte international relatif aux droits économiques, sociaux et culturels] et au PIDCP [Pacte international relatif aux droits civils et politiques], le paragraphe 2 de la résolution $1514(\mathrm{XV})$ de l'Assemblée générale de l'ONU et le titre VII de l'acte final d'Helsinki de $1975^{121}$.

De cette constatation découle une obligation internationale pour l'UE de ne pas reconnaître une annexion illégale:

212. Étant donné que l'affirmation de la souveraineté marocaine sur le Sahara occidental résulte d'une violation du droit du peuple de ce territoire à l'autodétermination pour les raisons que j'ai évoquées aux points 147 à 186 des présentes conclusions, l'Union a manqué à son obligation de ne pas reconnaître la situation illicite découlant de la violation du droit du peuple du Sahara occidental à l'autodétermination par le Royaume du Maroc, ainsi qu'à celle de ne pas prêter aide ou assistance au maintien de cette situation (187). De ce fait, dans la mesure où ils s'appliquent au territoire du Sahara occidental et aux eaux y adjacentes, l'accord de pêche et le protocole de 2013 sont incompatibles avec l'article 3, paragraphe 5, TUE, l'article 21, paragraphe 1, premier alinéa, TUE, l'article 21, paragraphe 2, sous b) et c), TUE, et les articles 23 TUE et 205 TFUE, qui imposent à l'Union l'obligation que son action extérieure protège les droits de l'homme et respecte strictement le droit international ${ }^{122}$.

L'avocat général rejette qu'un prétendu statut de "puissance administrante de facto » (rappelons que cette pseudo-catégorie fut employée par le service juridique du PE), qui n'existe pas en droit international, puisse justifier l'exploitation des eaux du Sahara occidental ${ }^{123}$ ). De plus, à son avis, le droit international humanitaire est applicable car le conflit qui a opposé le Maroc et le Front Polisario était un conflit armé au sens de la Convention IV de Genève et la présence du Maroc sur la partie du territoire sous son contrôle s'analyse en une occupation $^{124}$. Cependant, ce droit n'a pas été appliqué parce que le Maroc qualifie sa présence comme «souveraine» et non «occupante». Étant donné que pour l'avocat général les accords de pêche s'appliquent au Sahara occidental, il propose son annulation en ce qui concerne le Sahara occidental:

285. Par conséquent, je considère que les dispositions de l'accord de pêche et le protocole de 2013 ne donnent 
aucune garantie que l'exploitation halieutique des eaux adjacentes au Sahara occidental se fasse pour le bénéfice du peuple de ce territoire. En ce sens, les actes contestés ne respectent ni le principe de souveraineté permanente sur les ressources naturelles (268), ni l'article 55 du règlement de La Haye de 1907, ni l'obligation de l'Union de ne pas reconnaître une situation illicite découlant de la violation de ces dispositions et de ne pas prêter aide ou assistance au maintien de cette situation.

[...]

293. Il découle de ce qui précède que les actes contestés, qui sont applicables au territoire du Sahara occidental et aux eaux y adjacentes en ce qu'ils relèvent de la souveraineté ou de la juridiction du Royaume du Maroc, violent l'obligation de l'Union de respecter le droit du peuple de ce territoire à l'autodétermination ainsi que son obligation de ne pas reconnaître une situation illégale découlant d'une violation de ce droit et de ne pas prêter aide ou assistance au maintien de cette situation. De plus, en ce qui concerne l'exploitation des ressources naturelles du Sahara occidental, les actes contestés ne mettent pas en place les garanties nécessaires pour assurer que cette exploitation se fasse au bénéfice du peuple de ce territoire ${ }^{125}$.

\section{L'arrêt de la grande chambre de la CJUE du 27 février 2018}

La grande chambre de la CJUE a rendu son arrêt sur l'affaire Western Sahara Campaign le 27 février $2018^{126}$, date fortement symbolique puisque la République arabe sahraouie démocratique fut proclamée le 27 février 1976. La Cour a préféré réitérer sa doctrine précédente (affaire C-104/16 P) et n'a pas voulu partager la prémisse des conclusions de l'avocat général. La question fondamentale était donc de juger si les accords étaient "applicables» au Sahara occidental et non s'ils étaient «appliqués » à ce territoire.

La CJUE part du principe que le droit international, et notamment le principe d'autodétermination, doivent être respectés par le droit européen:

63. En effet, l'inclusion du territoire du Sahara occidental dans le champ d'application de l'accord d'association enfreindrait certaines règles de droit international général applicables dans les relations entre l'Union et le Royaume du Maroc, à savoir le principe d'autodétermination, rappelé à l'article $1^{\text {er }}$ de la charte des Nations unies, et le principe de l'effet relatif des traités, dont l'article 34 de la convention de Vienne sur le droit des traités constitue une expression particulière (arrêt du 21 décembre 2016, Conseil/ Front Polisario, C-104/16 P, EU:C:2016:973, points 88 à 93, 100, 103 à 107 et 123$)^{127}$.

La CJUE a choisi d'interpréter la teneur de la clause «eaux relevant de la souveraineté ou de la juridiction» (du Maroc) contenue dans les accords en appliquant un raisonnement clair:

68. Il en découle que les eaux sur lesquelles l'État côtier est en droit d'exercer une souveraineté ou une juridiction, en vertu de la convention sur le droit de la mer, se limitent aux seules eaux adjacentes à son territoire et relevant de sa mer territoriale ou de sa zone économique exclusive.

69. Par voie de conséquence, et compte tenu du fait que le territoire du Sahara occidental ne fait pas partie du territoire du Royaume du Maroc, ainsi que cela a été rappelé aux points 62 à 64 du présent arrêt, les eaux adjacentes au territoire du Sahara occidental ne relèvent pas de la zone de pêche marocaine visée à l'article 2, sous a), de l'accord de partenariat ${ }^{128}$.

De cette manière, la Cour rejette la thèse avancée par quelques auteurs qui considéraient que l'expression «eaux relevant de la souveraineté ou de la juridiction » (du Maroc) permettait l'inclusion des eaux du Sahara occidental ${ }^{129}$. Cette thèse fut contestée par un autre auteur avec des arguments retenus par la Cour dans cet arrêt ${ }^{130}$.

Mais si la Cour n'a pas suivi la prémisse de l'avocat général, elle le suit pour exclure qu'une qualification des eaux du Sahara occidental comme relevant d'une supposée «souveraineté» ou «juridiction» (sous la justification d'un prétendu statut de "puissance administrante de iure» ${ }^{131}$, de «puissance administrante de facto» ou même comme «puissance occupante ${ }^{132}$ ) du Maroc puisse servir de base juridique pour un éventuel accord de pêche UE-Maroc incluant les eaux du Sahara occidental:

71. Cependant, s'agissant de l'expression « eaux relevant de la souveraineté $[\ldots]$ du Royaume du Maroc» employée à l'article 2, sous a), de l'accord de partenariat, il doit être relevé qu'il serait contraire aux règles de droit international visées au point 63 du présent arrêt, que l'Union doit respecter et qui s'appliquent mutatis mutandis en l'occurrence, d'inclure, à ce titre, les eaux directement adjacentes à la

125. Ibid.

126. CJUE, arrêt de la Cour (GC), 27 février 2018, Western Sahara Campaign, affaire C-266/16.

127. Ibid.

128. Ibid.

129. Voir, par exemple, J. Soroeta Liceras, «La posicíon de la Unión Europea en el conflicto del Sahara Occidental, una muestra palpable (más) de la primacía de sus intereses económicos y políticos sobre la promoción de la democracia y de los derechos humanos", Revista de Derecho Comunitario Europeo, $\mathrm{n}^{\circ}$ 34, 2009, p. 823-864, spéc. p. 835 .

130. C. Ruiz Miguel, «La Unión Europea y el Sahara Occidental...», p. 178-179.

131. Voir C. Ruiz Miguel, «El acuerdo de pesca UE-Marruecos o el intento español de considerar a Marruecos como "potencia administradora” del Sahara Occidental», Anuario Español de Derecho Internacional, n 22, 2006, p. 395-412.

132. Voir E. Milano, «The New Fisheries Partnership Agreement between the European Community and the Kingdom of Morocco: Fishing too South?", Anuario Español de Derecho Internacional, n 22, 2006, p. 413-457; M. Dawidowicz, "Trading Fish or Human Rights in Western Sahara? Self-Determination, Non-Recognition and the EC-Morocco Fisheries Agreement ", in Statehood, Self-Determination: Reconciling Tradition and Modernity in International Law, D. French (dir.), Cambridge, Cambridge University Press, 2013, p. 250-276; B. Saul, «The Status of Western Sahara as Occupied Territory under International Humanitarian Law and the Exploitation of Natural Ressources ", Global Change, Peace and Security, vol. 27, n 3, 2015, p. 301-322, Sydney Law School Legal Studies Research Paper, nº 15/81, septembre 2015 
côte du territoire du Sahara occidental dans le champ d'application de cet accord. En conséquence, l'Union ne saurait valablement partager une intention du Royaume du Maroc d'inclure, à un tel titre, les eaux en question dans le champ d'application dudit accord.

72. Quant à l'expression «eaux relevant de [...] la juridiction du Royaume du Maroc» figurant à cette disposition, le Conseil et la Commission ont envisagé, entre autres hypothèses, que le Royaume du Maroc puisse être regardé comme une "puissance administrante de facto " ou une puissance occupante du territoire du Sahara occidental et qu'une telle qualification puisse s'avérer pertinente en vue de déterminer le champ d'application de l'accord de partenariat. À cet égard, il suffit toutefois d'observer que, sans même qu'il soit besoin d'examiner si une éventuelle intention commune des parties à l'accord de partenariat de donner à cette expression un sens particulier, afin de tenir compte de telles circonstances, aurait été conforme aux règles de droit international qui lient l'Union, une telle intention commune ne saurait, en tout état de cause, être constatée en l'occurrence dès lors que le Royaume du Maroc a catégoriquement exclu d'être une puissance occupante ou une puissance administrante du territoire du Sahara occidental.

73. Il résulte de l'ensemble des considérations qui précèdent que les eaux adjacentes au territoire du Sahara occidental ne relèvent pas de l'expression « eaux relevant de la souveraineté ou de la juridiction du Royaume du Maroc », figurant à l'article 2, sous a), de l'accord de partenariat ${ }^{133}$

En conclusion, la Cour a tranché l'affaire, comme elle l'avait fait en 2016, sur la base des principes du droit international, notamment ceux qui régissent le statut du territoire du Sahara occidental, ce qui ne rend pas nécessaire l'usage des arguments relevant des droits fondamentaux.

\section{Le Conseil récidive alors que la Cour enfonce le clou}

Le jour où la Cour a publié son arrêt, la haute représentante de l'Union pour les affaires étrangères et la politique de sécurité et vice-présidente de la Commission européenne, Federica Mogherini, et le ministre des Affaires étrangères et de la coopération du Royaume du Maroc, Nasser Bourita, ont signé une déclaration conjointe précisant que

Les deux parties restent déterminées à préserver leur coopération dans le domaine halieutique. À cet égard, elles expriment leur volonté de négocier les instruments nécessaires relatifs au partenariat halieutique ${ }^{134}$.

Et, de fait, trois semaines plus tard le Conseil a approuvé une recommandation autorisant la Commission à ouvrir des négociations avec le Maroc pour conclure un nouveau protocole de pêche ${ }^{135}$. La Commission propose de négocier une modification de l'accord de partenariat dans le secteur de la pêche (APP) entre l'UE et le Royaume du Maroc et de conclure un protocole mettant en œuvre cet accord. Cette proposition est présentée à la suite de l'arrêt rendu le 27 février 2018 par la CJUE dans l'affaire C-266/16 par laquelle la Cour juge que les eaux adjacentes au territoire du Sahara occidental ne font pas partie de la zone de pêche visée dans l'accord de pêche.

Une fois connu l'arrêt de la Cour on s'attendait à ce que, devant une aussi claire doctrine, le Conseil s'en tienne au respect de la décision judiciaire. Mais le Conseil récidive. Selon le "Contexte de la proposition" incluse dans l' «Exposé des motifs» de la Recommandation de décision:

La position de l'Union est qu'il est possible d'étendre les accords bilatéraux avec le Maroc au Sahara occidental sous certaines conditions. Toutefois, il est entendu que tout accord ne sera que provisoire tant que la résolution $\mathrm{du}$ conflit dans le cadre des Nations unies et conformément aux résolutions pertinentes du Conseil de sécurité des Nations unies restera pendante ${ }^{136}$.

Une telle affirmation est surprenante, étant donné que la Cour a clairement indiqué que, les eaux adjacentes à la côte du Sahara occidental ne faisant pas partie du Maroc, elles ne peuvent faire partie d'un accord entre l'UE et le Maroc $^{137}$. Par ailleurs, le Conseil méprise les résolutions de l'Assemblée générale dont certaines ont elles aussi une force obligatoire et furent citées par la Cour, tout comme l'avis consultatif de la Cour internationale de justice de 1975.

Le préambule de la Recommandation de décision ajoute une étonnante affirmation:

133. CJUE, arrêt de la Cour (GC), 27 février 2018.

134. Déclaration conjointe par Federica Mogherini et le ministre des Affaires étrangères et de la coopération du Royaume du Maroc, Nasser Bourita, Bruxelles, 27 février 2018, en ligne: https://eeas.europa.eu/headquarters/headquarters-homepage/42477/node/42477_fr.

135. Recommandation de décision du Conseil autorisant la Commission à ouvrir des négociations au nom de l'Union européenne en vue de la modification de l'accord de partenariat dans le secteur de la pêche et de la conclusion d'un protocole avec le Royaume du Maroc, Bruxelles, 21 mars 2018, COM(2018) 151 final, en ligne: https://eur-lex.europa.eu/legal-content/FR/TXT/HTML/?uri=CELEX:52018PCo151. Les négociations sont effectivement lancées le 19 avril 2018 (https:/eeas.europa.eu/headquarters/headquarters-homepage/43193/lancement-des-négociations-pourun-accord-accord-de-partenariat-dans-le-secteur-de-la-pêche_en).

136. Ibid.

137. CJUE, arrêt de la Cour (GC), 27 février 2018, Western Sahara Campaign: «68. Il en découle que les eaux sur lesquelles l'État côtier est en droit d'exercer une souveraineté ou une juridiction, en vertu de la convention sur le droit de la mer, se limitent aux seules eaux adjacentes à son territoire et relevant de sa mer territoriale ou de sa zone économique exclusive./69. Par voie de conséquence, et compte tenu du fait que le territoire du Sahara occidental ne fait pas partie du territoire du Royaume du Maroc, ainsi que cela a été rappelé aux points 62 à 64 du présent arrêt, les eaux adjacentes au territoire du Sahara occidental ne relèvent pas de la zone de pêche marocaine visée à l'article 2, sous a), de l'accord de partenariat». 
[...] considérant que l'Union soutient les efforts déployés par les Nations unies pour trouver une solution politique mutuellement acceptable qui permettrait l'autodétermination de la population du Sahara occidental en conformité avec les principes et les objectifs de la charte des Nations unies $[\ldots]^{138}$.

L'affirmation est bel et bien étonnante parce que si le Conseil soutient, comme il le prétend, une solution «mutuellement acceptable» qui permettrait l'«autodétermination » de la "population» (sic) du Sahara occidental, une telle négociation sur les eaux du Sahara occidental devrait se dérouler avec les deux parties qui doivent accepter la « solution mutuellement acceptable» ce qui, de toute évidence, n'est pas le cas, l'autre partie au conflit, c'est-à-dire le Front Polisario, n'étant pas invitée à négocier. Si l'UE désire vraiment soutenir une « solution mutuellement acceptable» au conflit du Sahara occidental elle devrait accorder une solution qui soit elle aussi «mutuellement acceptable» pour la question de la pêche dans les eaux du Sahara occidental. Bien entendu, une telle solution devrait être en conformité avec les principes et propos de la Charte des Nations unies $^{139}$ et les normes impératives du droit international, notamment le principe de la souveraineté permanente sur les ressources naturelles des peuples. En réalité, pourquoi vouloir introduire dans l'accord « une clause de réexamen permettant de prendre en compte une solution politique mutuellement acceptable qui permettrait l'autodétermination de la population du Sahara occidental en conformité avec les principes et les objectifs de la charte des Nations unies ${ }^{140}$ ? Il semble que cette clause soit insérée pour s'adapter à un éventuel accord entre le Maroc et le Front Polisario établissant un cadre pour la gestion des ressources naturelles du territoire.

Les Directives de négociation pour la Commission incluent aussi une référence aux droits de l'homme. Dans ce document du mandat de négociation contenu dans l'annexe à la Recommandation de décision, on établit que les négociations doivent viser «à prévoir une clause relative aux conséquences des violations des droits de l'homme et des principes démocratiques". Il ne s'agit pas d'une référence particulière au Sahara occidental, car cela ressemble davantage à celle contenue dans l'accord d'association. Cependant, l'expérience dudit accord est tout à fait décevante. En effet, à la différence de ce qui s'est passé dans les accords UE-ACP, l'accord d'association
UE-Maroc prévoit un mécanisme consensuel de règlement des différends qui rend impossible l'adoption de sanctions pour les violations des droits de l'homme commises par le Maroc ${ }^{141}$.

La CJUE a tranché la question dans deux arrêts de la grande chambre (2016 et 2018) rigoureusement argumentés. La doctrine est claire et ne laisse aucun doute sur l'illicéité d'inclure le Sahara occidental dans les accords entre l'UE et le Maroc.

Le mandat approuvé par le Conseil pour négocier un nouveau protocole de pêche entre l'UE et le Maroc semble tout à fait opposé aux arrêts de la CJUE. La prétention d'inclure les droits fondamentaux comme critère d'évaluation de l'éventuel protocole n'est qu'un écran de fumée visant à cacher une initiative illégale. Les droits de l'homme sont une partie du droit interne et international, qui n'a pas été prévue pour affaiblir les normes obligatoires du droit international et du droit européen. Certes importants, ils ne peuvent toutefois être instrumentalisés pour bafouer le droit international et le droit européen qui établissent l'obligation de les respecter.

Le 20 juillet 2018, la Commission a publié une déclaration conjointe de l'UE et du Royaume du Maroc annonçant la conclusion d'un nouvel accord de partenariat dans le secteur de la pêche ${ }^{142}$. Quelques jours après cette déclaration, la Commission a publié une dépêche précisant que

La Commission européenne s'engage pleinement à s'assurer que le présent accord bénéficiera à toutes les populations, y compris celle du Sahara occidental. Conformément au mandat de négociation et compte tenu des arrêts de la Cour de Justice européenne portant sur les accords entre l'UE et le Maroc, le texte qui est négocié prévoit dès lors des dispositions strictes portant sur la répartition géographique et sociale de ces bénéfices ${ }^{143}$.

Mais, alors que la Commission s'obstinait à signer cet accord, la Cour lui a envoyé un troisième avertissement. Le 19 juillet (un jour avant la signature du nouvel accord de pêche), la Cour a rendu une ordonnance qui déclare irrecevable une demande du Front Polisario contre le protocole fixant les possibilités de pêche prévues par l'accord de partenariat entre l'Union et le Royaume du Maroc dans le secteur de la pêche, avec l'argument, une fois de plus réitéré, que le Sahara occidental ne fait pas partie du Maroc; les eaux adjacentes au territoire du

138. Recommandation de décision du Conseil..., 21 mars 2018.

139. Dans les Directives de négociation contenues en annexe à la Recommandation de décision (COM(2018) 151 final), les négociations de la Commission doivent viser, parmi d'autres, l'objectif de: «[...] soutenir les efforts déployés par le secrétaire général des Nations unies pour trouver une solution permettant l'autodétermination de la population du Sahara occidental en conformité avec les principes et les objectifs de la charte des Nations unies».

140. Directives de négociation contenues en annexe à la Recommandation de décision (COM(2018) 151 final).

141. Voir C. Ruiz Miguel, «La Unión Europea y el Sahara Occidental... », p. 191-193.

142. Déclaration conjointe de l'UE et du Royaume du Maroc sur la fin des négociations pour un nouvel accord dans le secteur de la pêche, 20 juillet 2018 STATEMENT/18/4630, en ligne: http://europa.eu/rapid/press-release_STATEMENT-18-4630_fr.htm.

143. «Maroc-Union européenne: fin des négociations pour un nouvel accord dans le secteur de la pêche», 25 juillet 2018, en ligne: https://ec.europa.eu/ fisheries/maroc-union-européenne-fin-des-négociations-pour-un-nouvel-accord-dans-le-secteur-de-la-pêche_en. 
Sahara occidental ne relèvent en effet pas des eaux sous juridiction marocaine ${ }^{144}$. La Cour veut être claire:

[...] il serait contraire aux règles de droit international [...], que l'Union doit respecter et qui s'appliquent mutatis mutandis en l'occurrence, d'inclure dans le champ d'application de l'accord de partenariat les eaux directement adjacentes à la côte du territoire du Sahara occidental au titre d'eaux relevant de la souveraineté du Royaume du Maroc. En conséquence, l'Union ne saurait valablement partager une intention du Royaume du Maroc d'inclure, à un tel titre, les eaux en question dans le champ d'application dudit accord ${ }^{145}$.

Le Conseil et le Parlement européen persisteront-ils à braver la Cour?

144. CJUE, ordonnance du tribunal ( $5^{\mathrm{e}}$ chambre élargie), 19 juillet $2018, \$ 50$, en ligne: http://curia.europa.eu/juris/document/document_print.jsf? doclang $=$ FR\&text $=$ \&pageIndex $=0$ \&part $=1 \&$ mode $=$ req\&docid $=204281 \& 0 c c=$ first $\&$ dir $=\& c i d=200066$. 145. Ibid., $\$ 48$. 